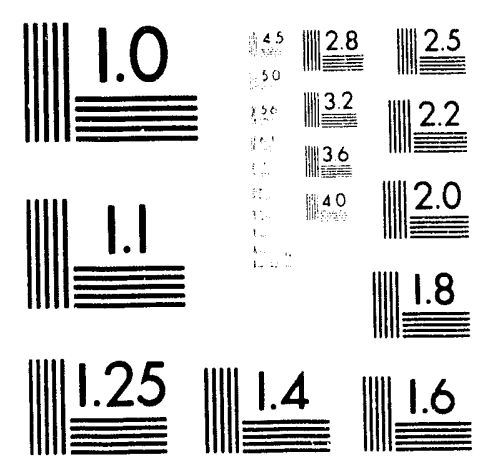



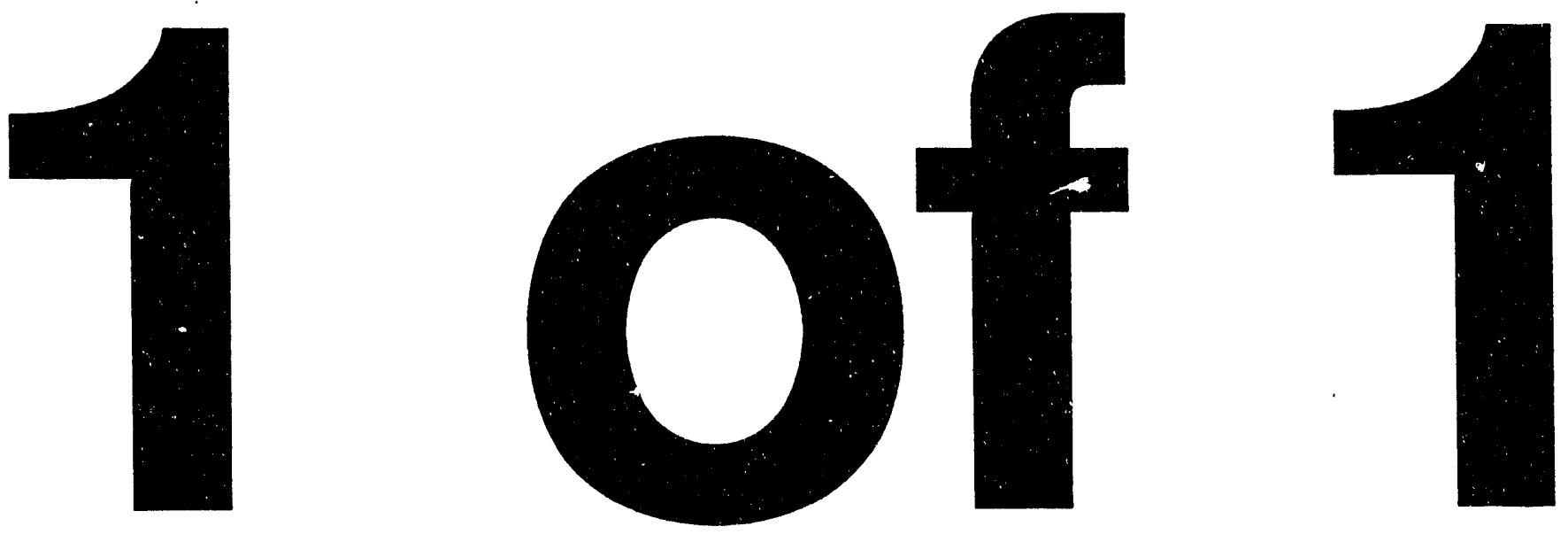


\title{
Direct Detection of Atomic Ions from Molerular Photofragmentation during Nonresonant Multiphoton Ionization of Sputtered Species*
}

\author{
S.R. Coon, W.F. Calaway, M.J. Pellin \\ Materials Science, Chemical Technology, and Chemistry Divisions \\ Argonne National Laboratory, Argonne, IL 60439 \\ J.W. Burnett \\ Ames Laboratory, Ames, IA $500 ! 1$
}

and

J.M. White

Department of Chemistry

University of Texas at Austin, Austin, TX 78712

\section{DISCLAIMER}

\begin{abstract}
This report was prepared as an account of work sponsored by an agency of the United States Government. Neither the United States Government nor any age..iy thereof, nor any of their employees, makes any warranty, express or implied, or assumes any legal liability or responsibility for the accuracy, completeness, or usefulness of any information, apparatus, product, or process disclosed, or represents that its use would not infringe privately owned rights. Reference herein to any specific commercial product, process, or service by trade name, trademark, manufacturer, or otherwise does not necessarily constitute or imply its endorsement, recommendation, or favoring by the United States Government or any agency thereof. The views and opinions of authors expressed herein do not necessarily state or reflect those of the United States Government or any agency thereof.
\end{abstract}

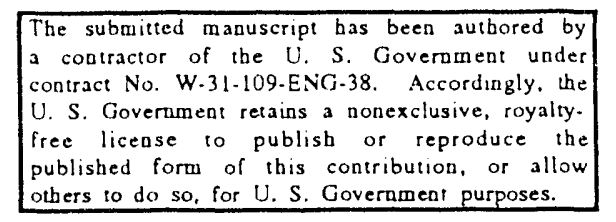

*Work supported by the U.S. Department of Energy, BES-Materials Sciences, under Contract W-31-109-ENG-38. 


\title{
Direct Detection of Atomic Ions from Molecular Photofragmentation during Nonresonant Multiphoton Ionization of Sputtered Species
}

\author{
S.R. Coon*, W.F. Calaway, M.J. Pellin \\ Materials Science, Chemical Technology, and Chemistry Divisions \\ Argonne National Laboratory, Argonne, IL 60439 \\ J.W. Burnett \\ Ames Laboratory, Ames, IA 50011 \\ and \\ J.M. White \\ Department of Chemistry \\ University of Texas at Austin, Austin, TX 78712
}

\begin{abstract}
The photoionization of sputtered $\mathrm{Cu}, \mathrm{Al}$, and $\mathrm{Ru}$ atoms and dimers was investigated by measuring velocity distributions using both resonant and nonresonant photoionization. Nonresonant ionization produced an atomic distribution that peaked at the same velocity as the respective dimer distribution, indicating that virtually all the nonresonant atomic ion signal is from photofragmented dimers. Various mechanisms of dimer photofragmentation are discussed. Domination of the atomic photoion channel by molecule fragmentation appears to be a general phenomenon that must be accounted for in all gas-phase multiphoton nonresonant ionization experiments at easily achievable laser power densities $\left(\leq 10^{9} \mathrm{~W} / \mathrm{cm}^{2}\right)$.
\end{abstract}

* Graduate Research Assistant. Department of Chemistry, University of Texas at Austin and Laboratory Graduate Participant, Argonne National Laboratory 


\section{Introduction}

Laser photoionization has become an important tool in the detection of neutral species, e.g., those ejected from a surface by sputtering or laser ablation. Its power lies in the potential to achieve saturation (100\% ionization) of atomic species either by including resonant transitions in the ionization scheme or by using very high nonresonant laser power density. Saturation is desired in order to make quantitative determinations of the concentrations of species in the gas phase without knowledge of their ionization cross sections. The use of resonant transitions greatly reduces the power needed to reach saturation, but has the drawback that resonant ionization is very specific, requiring precise photon wavelengths to ionize each element. Thus, the number of elements that may be resonantly ionized at one time depends on the number of lasers available. The appeal of nonresonant ionization is that all elements can be ionized simultaneously and detected quantitatively if the power density of the laser can be made large enough to reach saturation. Lasers capable of producing the necessary power density are excimer lasers operated with $\mathrm{XeCl}, \mathrm{KrF}$, or ArF gas mixtures and $\mathrm{YAG}$ lasers. However, the ionization potential of nearly every element is above some or all of these photon energies, so that at least two-photon nonresonant excitation is generally needed to achieve ionization. The power density necessary to saturate a two-photon nonresonant ionization process has been measured to be $10^{10-11} \mathrm{~W} / \mathrm{cm}^{2}[1]$. Because of the divergent quality of excimer laser beams, this power density can be difficult to achieve. Below saturation intensity, not only is the ionization process nonquantitative due to differences in the multiphoton ionization cross sections of different species, 
but the atomic ions observed may not be from atoms, but from photofragmentation of molecules. Sin e molecular species are often in the gas phase with the atomic species, particularly in the case of sputtering or laser ablation, [2-11] photofragmentation of these species can contribute to, and even dominate, the atomic ion signal.

Photofragmentation of sputtered diatomics to atomic ions has been observed by Craig et al. [5] for In. They found, using resonant two-color two-photon ionization, that certain excited states of In atoms were not formed by the sputtering process, but by photofragmentation of $\operatorname{In}_{2}$. Kampwerth et al. [12] found during depth profiling of a $\mathrm{Pt} / \mathrm{PtSi} / \mathrm{Si}$ layered system that a substantial fraction of the $\mathrm{Si}$ photoion signal was due to

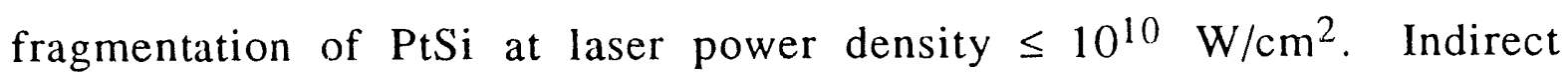
evidence has also been presented by Bryan et al. [13] for photofragmentation of molecules contributing to atomic ion signal (power density $\leq 10^{9} \mathrm{~W} / \mathrm{cm}^{2}$ ) in sputtering depth profile measurements of oxidized silver and oxidized silicon systems. Although these examples were once considered exceptional, recent results suggest that molecular photofragmentation to atomic ions is a quite general phenomenon. The data presented in this paper for $\mathrm{Cu}, \mathrm{Al}$ and $\mathrm{Ku}$ demonstrates that molecule photofragmentation can be the dominant source of atomic ion signal in experiments in which easily attainable nonresonant laser power densities are used to ionize neutral species. 


\section{Experimental}

The laser postionization time-of-flight technique used to measure velocity distributions of sputtered neutrals has been described previously $[2,3]$. Briefly, an ion pulse (200 ns) strikes a target causing ejection of atoms and clusters. Fixed velocity neutrals are selected by photoionizing the sputtered species in a laser volume a known distance, $r$, from the target at a known time, $t$, after the ion pulse. The velocity selected photoionized species are then mass separated using a time-offlight mass spectrometer. The signal for a given species ( $\propto$ number of that species in the laser volume) is then expressed as a function of velocity $(v=r / t)$. As shown in refs. [3] and [14], the velocity distribution for laser postionization experiments can be accurately approximated by

$$
\text { Signal } \propto v f(v)
$$

where $f(v)$ is the flux distribution of the species of interest. Eq. 1 is valid for any flux distribution, and as such is a very useful expression that has been largely overlooked.

Various resonant and nonresonant photoionization schemes have been employed in our experiments and are shown in Table 1. Three excimer gas mixtures, $\mathrm{XeCl}(308 \mathrm{~nm}, 4.0 \mathrm{eV}), \mathrm{KrF}(248 \mathrm{~nm}, 5.0 \mathrm{eV})$, and ArF $(193 \mathrm{~nm}, 6.4 \mathrm{eV})$ were used to produce fixed-frequency UV radiation, typica'ly for multiphoton nonresonant ionization. However, two of the excimer wavelengths (308 $\mathrm{nm}$ and $193 \mathrm{~nm}$ ) are coincidentally resonant with $\mathrm{Al}$ atomic transitions, and were used for resonant ionization of $\mathrm{Al}$ atoms. In addition, the $\mathrm{XeCl}$ excimer was used to pump a dye laser containing either DCM dye, the output of which was doubled to produce 
tunable light for $\mathrm{Cu}$ resonant ionization, or Exalite 376, which produced tunable light for $\mathrm{Ru}$ resonant ionization.

Two-laser experiments of the pump/probe type were also performed to investigate the mechanisms of atomic ion formation from diatomics. The probe laser beam was the same size and distance from the target that were typically used to measure velocity distributions ( $1 \mathrm{~mm}$ wide, $6 \mathrm{~mm}$ away). The pump laser beam was much larger and fully encompassed the space occupied by the probe laser plus a substantial portion of the volume between the probe laser and the target. Since the pump laser fires approximately $450 \mathrm{~ns}$ before the probe laser, the pump beam must be large enough to account for travel of the neutrals during that time interval. The pump laser was a $\mathrm{XeCl}$ excimer which was telescoped with $500 \mathrm{~mm}$ and $150 \mathrm{~mm}$ spherical lenses to achieve a maximum power density of about $10^{7} \mathrm{~W} / \mathrm{cm}^{2}$. The probe laser was an unfocussed ArF excimer, shaped with knife edges to produce a power density of approximately $10^{5} \mathrm{~W} / \mathrm{cm}^{2}$.

\section{Results and Discussion}

Determining the contribution of molecular photofragmentation to the atomic ion signal is not trivial. In mass spectrometry, the ions produced by photofragmentation are indistinguishable from the directly ionized species of the same mass. The dominance of photofragmentation over atomic ionization only became apparent when we measured the velocity distributions of sputtered neutral atoms from metal targets. This velocity distribution is known to be of the form[15-17] 


$$
f(v) \propto \frac{\left(\frac{v}{v_{b}}\right)^{3}}{\left(\left(\frac{v}{v_{b}}\right)^{2}+1\right)^{3}}
$$

where $v$ is the velocity of the atoms and $v_{b}$ is the velocity calculated from the surface binding energy, $E_{b}$, customarily taken to be the heat of sublimation.

Fig. 1 shows two velocity distributions of sputtered $\mathrm{Cu}$ atoms obtained with two-color two-photon ionization (327.4 nm plus $308 \mathrm{~nm}$ ). The two sets of data were measured with the dye laser tuned either on or off resonance, but otherwise under the same experimental conditions. The off-resonance signal $(\lambda=327.2 \mathrm{~nm})$ was about $1 / 15$ as large as the onresonance signal $(\lambda=327.4 \mathrm{~nm})$. T... solid line in the plot is the theoretical sputtered atom distribution according to eqs. 1 and 2, with $\mathrm{v}_{\mathrm{b}}=3.26 \mathrm{~km} / \mathrm{s}$. This pair of distributions illustrates the essential feature seen in all cases we have examined: The resonant distribution follows the theory reasonably well, but the nonresonant distribution does not. In particular, in the nonresonant distribution, the peak valocity and the relative intensity at high velocities are lower than predicted. The nonresonant velocity distributions obtained using only $193 \mathrm{~nm}$ or $308 \mathrm{~nm}$ light for photoionization peaked at the same velocity as the two-color nonresonant distribution.

Fig. 2 shows resonant $(193 \mathrm{~nm})$ and nonresonant $(248 \mathrm{~nm})$ velocity distributions for $\mathrm{Al}$ atoms. Although not shown, the velocity distribution measured using $308 \mathrm{~nm}$ resonant ionization tracked the $193 \mathrm{~nm}$ data. The solid line is the calculated (eqs. 1 and 2) distribution with $v_{b}=4.92 \mathrm{~km} / \mathrm{s}$. The dashed line is the measured one-photon ionized 
$(193 \mathrm{~nm}) \mathrm{Al}_{2}$ distribution, which is not expected to be significantly perturbed by fragmentation effects. Clearly, the nonresonant $\mathrm{Al}$ distribution follows the $\mathrm{Al}_{2}$ distribution. The nonresonant $\mathrm{Cu}$ and $\mathrm{Cu}_{2}$ distributions were also observed to track each nther. These results indicate that the atomic ions observed using nonresonant ionization are derived from dimers for both $\mathrm{Cu}$ and $\mathrm{Al}$. The nonresonant ionization of neutral atoms, the major species in the sputtered flux $[2,3,6,7,11]$, makes a negligible contribution to the atomic ion signal. In our experiments, the signal from neutral atoms dominated the dimer photofragmentation signal only when the atoms were resonantly ionized.

We also observed that, while resonantly ionized $\mathrm{Ru}$ atoms followed the predicted velocity distribution, the nonresonantly $(308 \mathrm{~nm})$ ionized distribution peaked at a lower velocity. It is likely that the parent species is $\mathrm{Ru}_{2}$, but the $\mathrm{Ru}_{2}{ }^{+}$intensity was too low to measure a velocity distribution in order to confirm this hypothesis. Very recent experiments on sputtered Sn [18] and $\mathrm{Ag}$ [11] two-photon ionized with $193 \mathrm{~nm}$ photons also suggest that the atoms detected are virtually all from photofragmented dimers.

We have shown that the dominance of the atomic ion signal by dimer photofragmentation is not rare, but is seen for several elements and ionization wavelengths in a moderate $\left(10^{9} \mathrm{~W} / \mathrm{cm}^{2}\right)$ power density regime. Since the fractional dimer sputtering yields from pure metals are on the order of 0.01 to 0.1 , the probability for two-photon nonresonant ionization of atoms must be at least two orders of magnitude smaller than the overall probability for the photofragmentation of diatomics to atomic ions at this power density. Clearly, at some larger laser power density, nonresonant ionization of atoms must eventually become the dominant source of atomic 
ion signal. While this suggests that strong focussing is the key to ionizing atoms more efficiently, volume effects due to the laser's spatial distribution and/or the acceptance volume of the mass spectrometer can lead to photofragmentation dominance up to very high power densities. This underscores the necessity of determining the origin of atomic ion signal in every multiphoton nonresonant photoionization experiment. The easiest way to insure reliable measurement of the neutral atom population is to resonantly ionize the atoms of interest. To minimize fragmentation when photoionizing molecules, one-photon ionization should be used.

Let us examine two mechanisms that can produce atomic ions from photoexcitation of neutral diatomics:

$$
\begin{array}{ll}
\begin{array}{l}
M_{2}+n \text { hv } \\
n=2,3
\end{array} & \text { dissociative ionization } \\
M_{2}+1 h v \rightarrow M^{*}+\mathrm{e}^{-}+M^{(*)} & \text { neutral dissociation } \\
M^{*}+1 h v \rightarrow M^{+}+e^{-} & \text {with subsequent ionization. }
\end{array}
$$

Dissociative ionization requires the absorption of energy (typically multiphoton) that exceeds the sum of the diatomic's dissociation energy and the atom's ionization potential. Generally, multiphoton absorption is easier for molecules than for atoms because the density of states for molecules is much higher. This could explain why photofragmentation of dimers dominates the atomic ion signal.

The neutral dissociation/ionization mechanism is another two-photon process that could explain our observations. In this mechanism, the first photon dissociates the molecule, and the second ionizes one or both neutral atoms. For neutral dissociation/ionization to dominate atomic nonresonant 
ionization, at least one of the atoms from the dissociated dimer must be in an excited state that is one-photon ionizable. Dissociation to ground state atoms will not result in the majority of atomic ions coming from diatomics, since these atoms will have the same ionization efficiency as atoms directly sputtered in the ground state.

One of the most interesting aspects of the neutral dissociation/ionization mechanism is that it is possible to distinguish the dissociation and ionization steps. Evidence, in the case of $\mathrm{Cu}$, is shown in fig. 3. Two excimer lasers were used in a pump/probe fashion. Fig. 3 shows the time-of-flight mass spectra of the ${ }^{63} \mathrm{Cu}$ and ${ }^{65} \mathrm{Cu}$ photoions created by the probe laser alone and the probe laser with the pump laser. Velocity distribution measurements have shown that all the atomic ion signals are from photofragmented dimers. Photoions created by the pump and the probe laser are extracted and detected at different times determined by the delay time between the two lasers. Neutrals that are not ionized by the pump laser remain in the ionization region and can be ionized by the probe laser. If dissociation followed by ionization were not important, the pump laser would deplete the available source of atomic ions, namely the copper dimers, and the probe signal would be smaller when the pump laser is added. The oppesite is seen in fig. 3. This can be explained by the second mechanism above. When the pump laser is on, neutral dissociation of copper dimers occurs, but either the excited state atoms that are formed are not one-photon ionizable by $308 \mathrm{~nm}$ or the dissociation takes longer than the duration of the laser pulse (20 ns). When the probe laser fires, the remaining excited state atoms can be onephoton ionized. Therefore, the pump laser dissociates the $\mathrm{Cu}_{2}$, producing atomic species that are easily ionized by the probe laser. While atomic 
ions are being produced from diatomics by both the pump laser and the probe laser alone, possibly by the dissociative ionization mechanism, the pump/probe experiments prove that neutral dissociation of copper dimers by $308 \mathrm{~nm}$ light does occur. A study of the photofragmentation of sputtered polyatomics will be included in a more detailed paper now in preparation.

\section{Conclusion}

The results of this investigation show that atomic photoion signals often do not represent the atomic population in sputtered fluxes. The parent species for an atomic ion signal can be identified by velocity distribution measurements of the atoms and molecules. The atomic ion signal in multiphoton nonresonant ionization of sputtered $\mathrm{Cu}, \mathrm{Al}$, and $\mathrm{Ru}$ comes from diatomic molecules when easily achievable laser power densities $\left(\leq 10^{9} \mathrm{~W} / \mathrm{cm}^{2}\right)$ are used. These results, combined with previously reported data on $\mathrm{In}, \mathrm{Sn}, \mathrm{Ag}$, and $\mathrm{Si}$ suggest that the production of atomic ions from diatomics is a general phenomenon that must be taken into account in all multiphoton nonresonant ionization experiments.

\section{Acknowledgements}

The authors would like to thank A. Wucher and W. Husinsky for making their results known to us prior to publication. S.R.C. is grateful for support from a National Science Foundation Graduate Fellowship and the 
Division of Educational Programs at Argonne National Laboratory. This work is supported by the U.S. Department of Energy, BES-Materials Sciences, under Contract W-31-109-ENG-38.

\section{References}

[1] S. Kaesdorf, H. Schröder and K. L. Kompa, Vacuum 41 (1990) 1669.

[2] S. R. Coon, W. F. Calaway, J. W. Burnett, M. J. Pellin, D. M. Gruen, D. R. Spiegel and J. M. White, Surf. Sci. 259 (1991) 275.

[3] S. R. Coon, W. F. Calaway, M. J. Pellin, G. A. Curlee and J. M. White, Nucl. Instrum. Methods Phys. Res. B, in press.

[4] S. R. Coon, W. F. Calaway, M. J. Pellin and J. M. White, Surf. Sci., submitted.

[5] B. I. Craig, J. P. Baxter, J. Singh, G. A. Schick, P. H. Kobrin, B. J. Garrison and N. Winograd, Phys. Rev. Lett. 57 (1986) 1351.

[6] W. Gerhard and H. Oechsner, Z. Physik B22 (1975) 41.

[7] J. Giber and W. O. Hofer, in: Proceedings of the Symposium on Sputtering, Eds. P. Varga, G. Betz and F. P. Viehböck (TU Wien, Vienna, 1980), p. 697.

[8] H. Gnaser and W. O. Hofer, App. Phys. A 48 (1989) 261.

[9] W. O. Hofer, in: Sputtering by Particle Bombardment III, Eds. R. Behrisch and K. Wittmaack, Topics in Applied Physics, Vol. 64 (Springer-Verlag, Berlin, 1991), p. 15.

[10] D. E. Powers, S. G. Hansen, M. E. Geusic, D. L. Michalopoulos and R. E. Smalley, J. Chem. Phys. 78 (1983) 2866.

[11] A. Wucher and M. Wahl, Nucl. Instrum. Methods Phys. Res. B, in press. 
[12] G. Kampwerth, M. Terhorst, E. Niehuis and A. Benninghoven, in: Secondary Ion Mass Spectrometry SIMS VIll, Eds. A. Benninghoven, K. T. F. Janssen, J. Tümpner and H. W. Werner (John Wiley \& Sons, Chichester, 1992), p. 563.

[13] S. R. Bryan, M. Nicholas and G. J. Havrilla, in: Secondary Ion Mass Spectrometry SIMS VIII, Eds. A. Benninghoven, K. T. F. Janssen, J. Tümpner and H. W. Werner (John Wiley \& Sons, Chichester, 1992), p. 571 .

[14] H. F. Arlinghaus, W. F. Calaway, C. E. Young, M. J. Pellin, D. M. Gruen and L. L. Chase, J. Appl. Phys. 65 (1989) 281.

[15] P. Sigmund, Phys. Rev. 184 (1969) 383.

[16] M. W. Thompson, Philos. Mag. 18 (1968) 377.

[17] H. Oechsner, Z. Physik 238 (1970) 433.

[18] W. Husinsky, G. Nicolussi and G. Betz, Nucl. Instrum. Methods Phys. Res. B, in press.

[19] V. Kaufman and W. C. Parkinson, J. Phys. Chem. Ref. Data 20 (1991) 775 .

[20] J. L. Kohl and W. H. Parkinson, Astrophys. J. 184 (1973) 641. 


\section{Table 1}

Photoionization schemes used in measuring velocity distributions.

\begin{tabular}{ccccc}
\hline Atom & $\begin{array}{c}\text { IP } \\
(\mathrm{eV})\end{array}$ & Wavelengths & $\begin{array}{c}\text { Power density } \\
\left(\mathrm{W} / \mathrm{cm}^{2}\right)\end{array}$ \\
\hline $\mathrm{Cu}$ & 7.7 & $327.4 \mathrm{~nm}\left(4 \mathrm{~s}^{2} \mathrm{~S}_{1 / 2} \rightarrow 4 \mathrm{p}^{2} \mathrm{P}_{3 / 2}\right)+308 \mathrm{~nm}$ & $10^{5}+10^{5-6}$ \\
& & $308 \mathrm{~nm}$ two-photon nonresonant & $10^{8}$ \\
& & $193 \mathrm{~nm} \mathrm{two-photon} \mathrm{nonresonant}$ & $10^{8}$ \\
$\mathrm{Al}$ & 6.0 & $193 \mathrm{~nm}\left(3 \mathrm{p}{ }^{2} \mathrm{P}_{1 / 2,3 / 2} \rightarrow 3 \mathrm{p}^{2}{ }^{2} \mathrm{~S}_{1 / 2}\right)[19,20]$ & $10^{2-5}$ \\
& & $308 \mathrm{~nm}\left(3 \mathrm{p}{ }^{2} \mathrm{P}_{1 / 2} \rightarrow 3 \mathrm{~d}^{2} \mathrm{D}_{3 / 2}\right)+308 \mathrm{~nm}$ & $10^{5}$ \\
& & $248 \mathrm{~nm} \mathrm{two-photon}$ nonresonant & $10^{8}$ \\
$\mathrm{Ru}$ & 7.4 & $372.8 \mathrm{~nm}\left(\mathrm{a}{ }^{5} \mathrm{~F}_{5} \rightarrow \mathrm{z}{ }^{5} \mathrm{~F}_{5}{ }^{0}\right)+308 \mathrm{~nm}$ & $10^{7}+10^{6-7}$ \\
& & $308 \mathrm{~nm} \mathrm{two-photon}$ nonresonant & $10^{6-7}$ \\
\hline
\end{tabular}


Figure Captions

Fig. 1 Velocity distributions of copper atoms measured with the $327.4 \mathrm{~nm}+308 \mathrm{~nm}$ photoionization scheme. Filled circles: dye laser tuned on resonance; Open circles: dye laser tuned $0.2 \mathrm{~nm}$ off resonance. Solid line: Theoretical at om distribution according to eqs. 1 and 2.

Fig. 2 Velocity distributions of aluminum atoms measured with: $193 \mathrm{~nm}$ one-photon resonant ionization (Filled circles); $248 \mathrm{~nm}$ two-photon nonresonant ionization (Open circles). Solid line: Theoretical atom distribution according to eqs. 1 and 2. Dashed line: $193 \mathrm{~nm}$ onephoton ionized $\mathrm{Al}_{2}$ velocity distribution.

Fig. 3 TOF mass spectra of sputtered copper atoms in pump/probe experiment. The ${ }^{63} \mathrm{Cu}$ and ${ }^{65} \mathrm{Cu}$ isotopes are shown for both the pump and the probe lasers. Solid line: probe laser only firing; Dashed line: pump and probe lasers firing. 


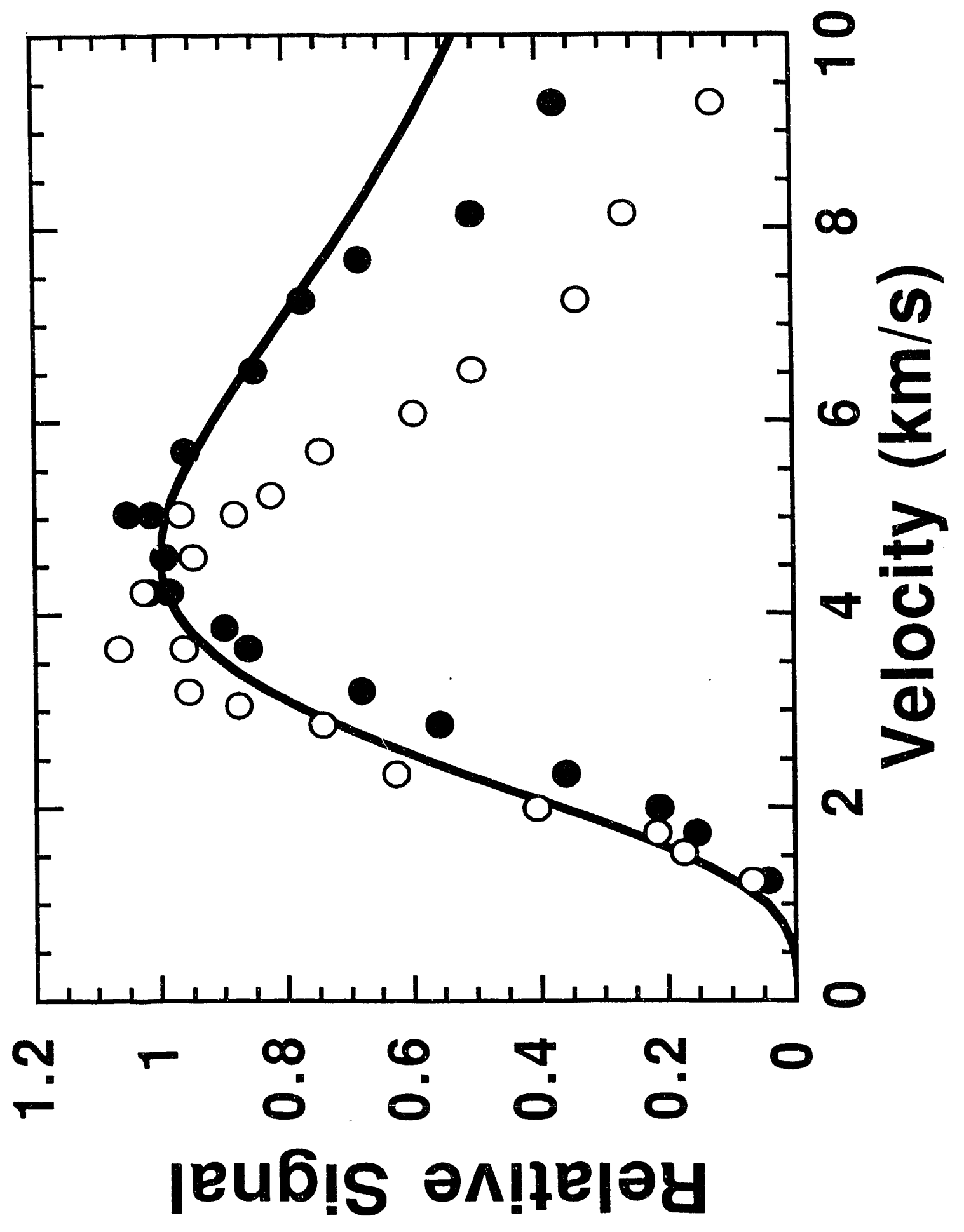




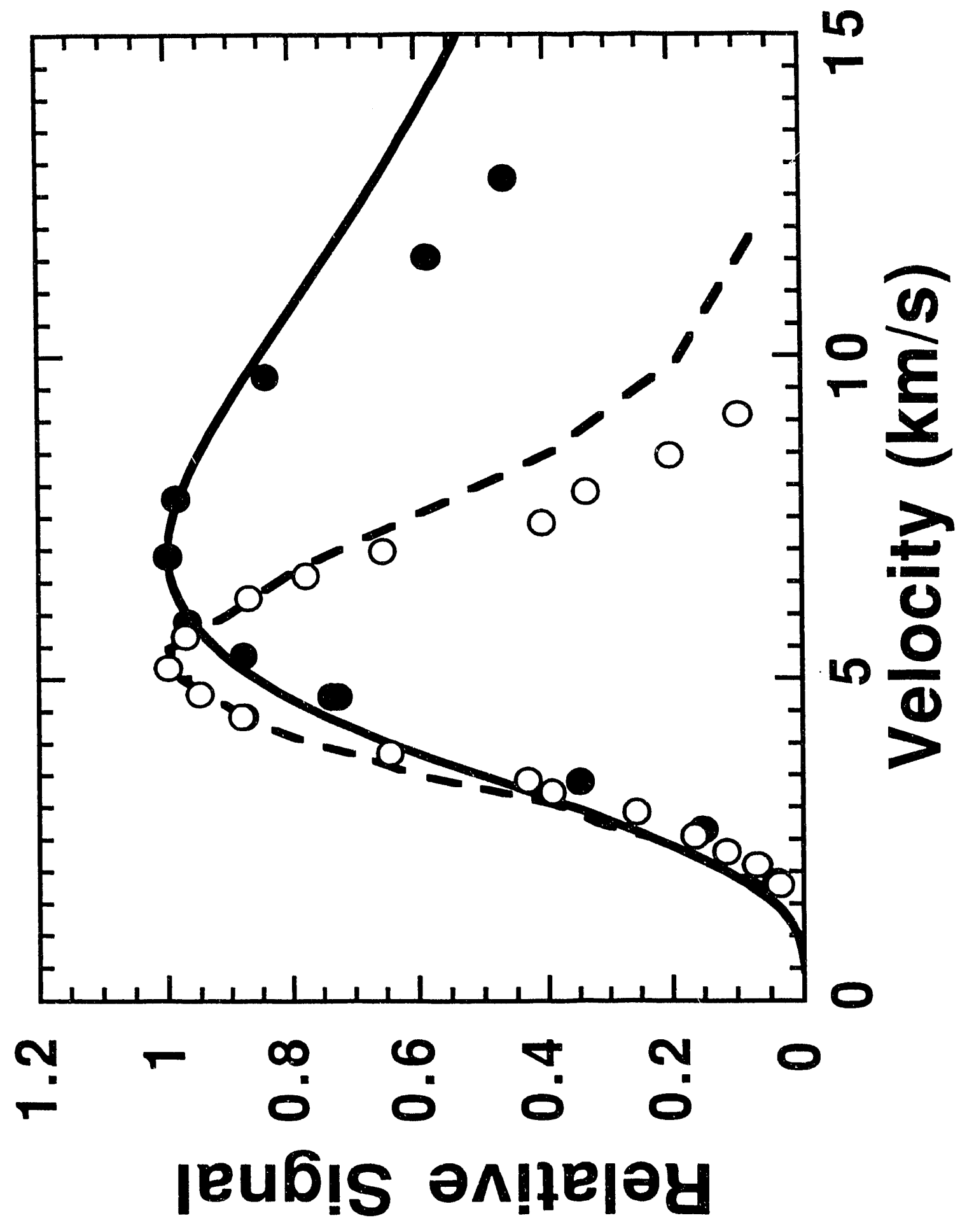




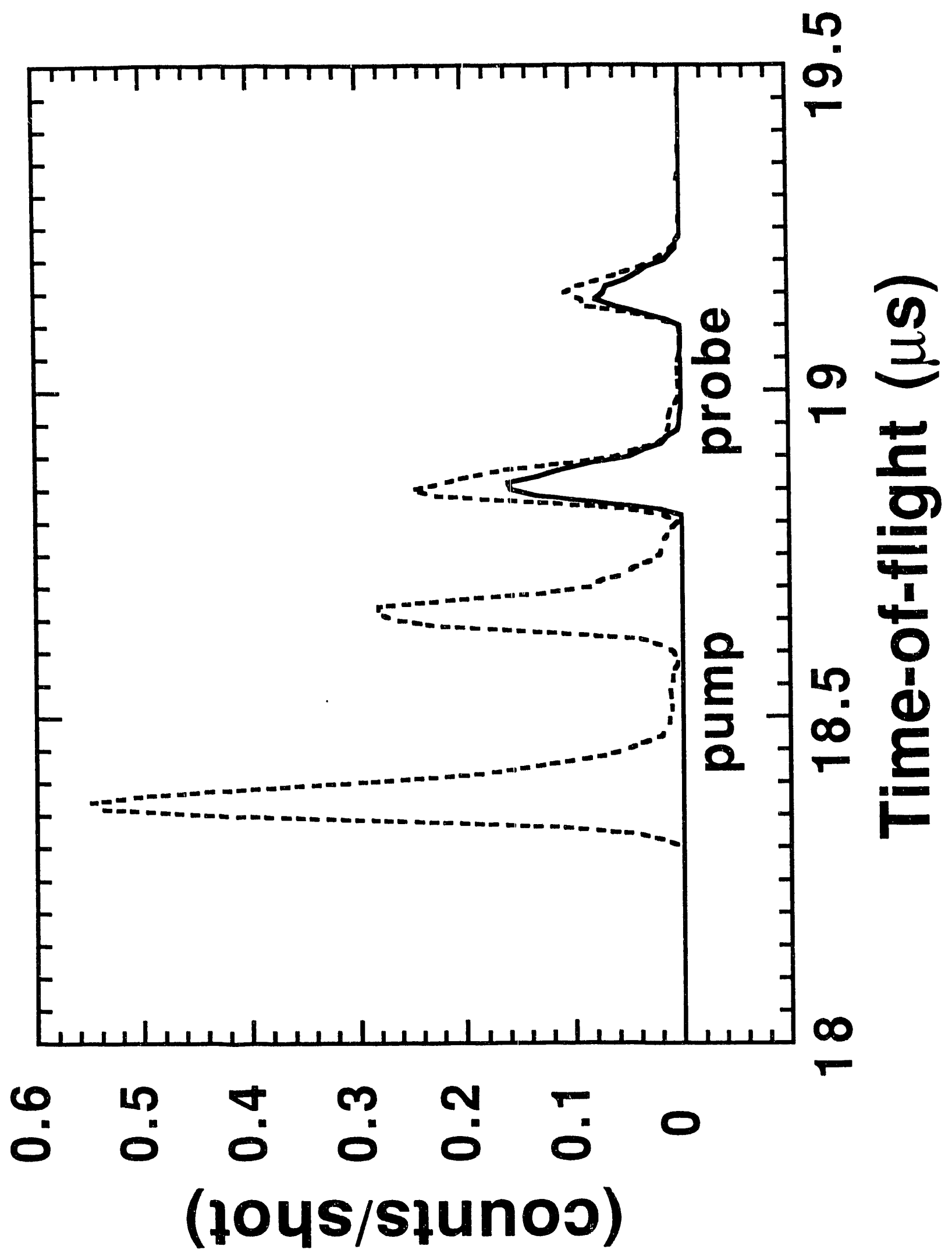



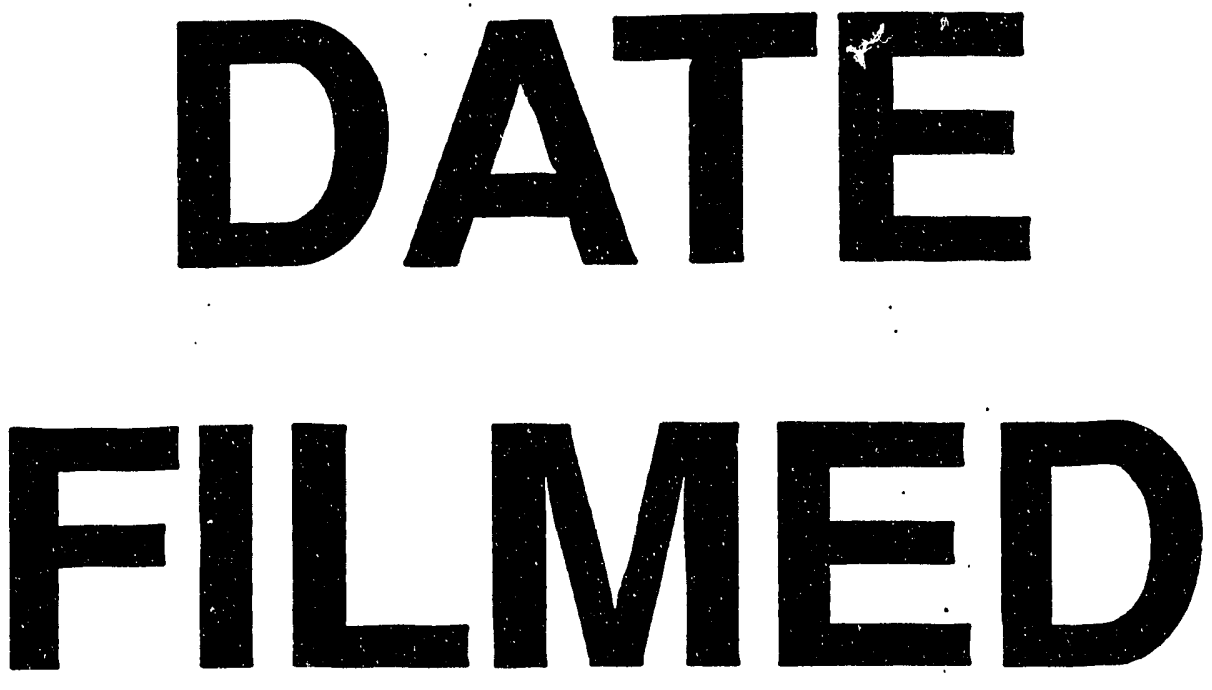

$11 / 17 / 93$
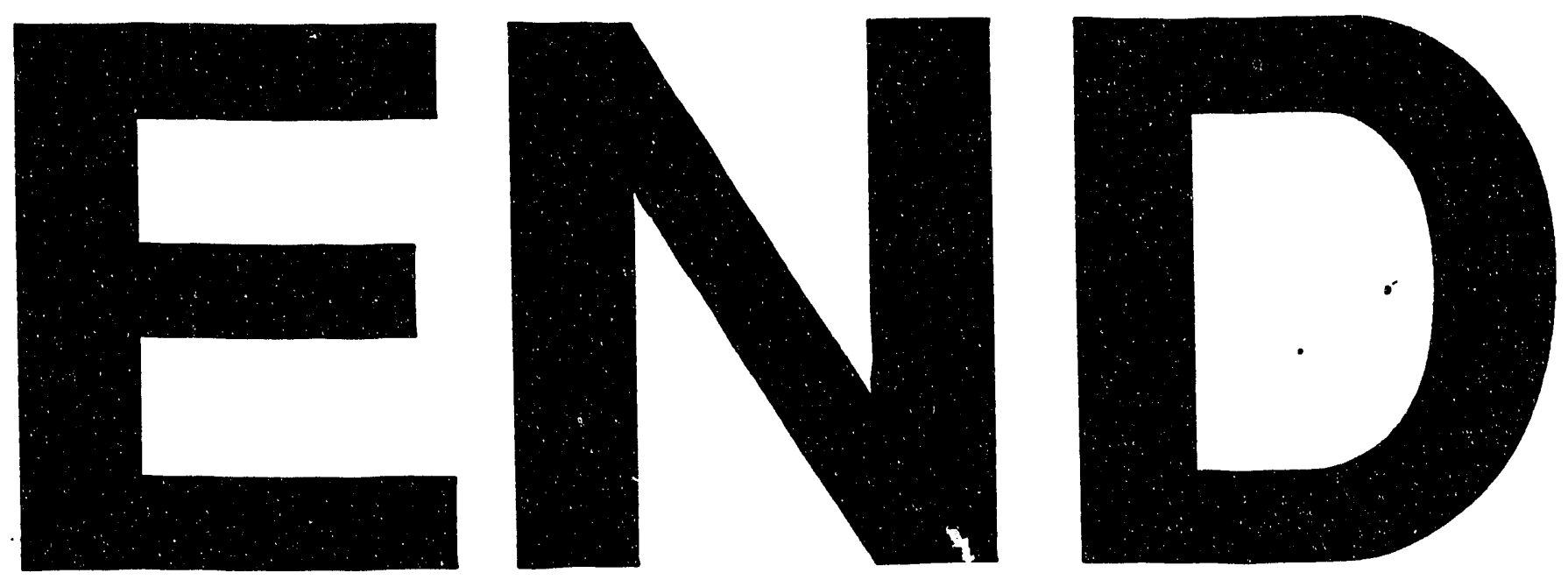
